\title{
LA FILOSOFÍA CHILENA DEL SIGLO XX COMO PENSAMIENTO CRÍTICO
}

\author{
Dr. Ricardo Salas A.*
}

Este artículo propone que es posible reconocer un eje central en la filosofía chilena del siglo XX ligada al desarrollo de la reflexión y la crítica. Mostrar esta posibilidad permitiría definir una historia de las ideas que tensiona de otro modo los imaginarios sociales y las ideas filosóficas en Chile. La hipótesis es que éstas no seguirían dinámicas propias -como pareciera sostener un cierto idealismo que estudia las ideas omitiendo sus contextos socio-históricos--, un mundo de las ideas no solo entendería el mundo, sino que también ofrecería pistas para actuar en el mundo. En definitiva, se busca distinguir entre los filósofos como intelectuales que generan pensamiento en sinergia con las tensiones de los mundos históricos, y los filósofos cuyo principal afán es devenir especialistas de textos canonizados por la institución universitaria y los curriculum de formación. El título La filosofía chilena del siglo XX como pensamiento crítico alude a esta distinción y pretende mostrar la existencia de un grupo relevante de filósofos chilenos que encontraron, por su particular apropiación de las circunstancias históricas que les tocó enfrentar, la especificidad misma de la génesis creativa de las ideas en que se articuló su pensamiento. Esta producción es parte de lo proponemos denominar una "historia auténtica del pensamiento crítico" y de la que Eduardo Molina, Jorge Millas y Humberto Giannini, entre otros, serían una parte importante.

Palabras clave: Filosofía chilena, pensamiento crítico, filosofía latinoamericana, historia de las ideas.

\section{THE 20TH CENTURY CHILEAN PHILOSOPHY AS CRITICAL THINKING}

This article proposes that is possible to recognize a main line in the 20th century Chilean philosophy linked to the development of reflection and critique. To show this possibility, would allow defining a history of ideas that tautens, in other way, the social imaginary and the philosophical ideas in Chile. The hypothesis is that these ideas wouldn't follow proper dynamics - as certain idealism that studies ideas omitting their socio-historical contexts seems to sustain-, a world of ideas not only would understand the world, but would also offer clues to act in the world. Ultimately it's seek to distinguish between philosophers as scholars who engender knowledge according to the historical world's tension, and the philosophers who's main goal is to become specialists in approved 
texts by academic institutions and training resumes. The title The 20th century Chilean philosophy as critical thinking alludes to the mentioned distinction and pretends to show the existence of a relevant group of Chilean philosophers that, by their particular appropriation of the historical circumstances they faced, found the very specificity in the creative genesis of the ideas that articulated their thought. This production is what we propose to name an "authentic history of critical thinking" in which Eduardo Molina, Jorge Millas and Humberto Giannini, among others, would be an important part of.

Keywords: Chilean philosophy, critical thinking, Latin American philosophy, history of ideas. 


\section{Introducción}

LA FILOSOFÍA CONTEMPORÁNEA HA RELEVADO EL PAPEL DE LA TEORÍA crítica que proviene de la Ilustración, del pensamiento crítico social del XIX, y que para otros se asocia a los aportes de los filósofos de la denominada "Escuela de Frankfurt", o al pensamiento neomarxista de la descolonización. Sin embargo, los estudios sistemáticos acerca de la historia de la filosofía en el siglo XX muestran que el pensamiento crítico es mucho más vasto que la teoría crítica europea; lo mismo se puede decir del pensar de los frankfurtianos que intentan elaborar un tipo de filosofìa articulada a las teorías sociológicas que dan cuenta de las transformaciones del marxismo en la sociedad moderna. Otra diferencia es que la teoría crítica frankfurtiana, a pesar de ser un pensamiento progresista, sigue siendo todavía un filosofar ligado a los contextos europeos. Hasta el momento, incluso en la última generación de filósofos, tales como Habermas y Honneth, no se encuentra una crítica de la sociedad capitalista que asuma lo que acontece en la periferias no-europeas ${ }^{1}$.

Por otra parte, en los últimos años se ha desarrollado un proceso creciente de toma de consciencia acerca de las formas diversas en que se desplegó el pensamiento crítico y alternativo en la historia de las ideas de América Latina ${ }^{2}$. Este tipo de pensar ya ha comenzado a ser rastreado y analizado por los estudiosos de la historia de las ideas. Sin embargo, es menester reconocer que el pensamiento emancipador y crítico no es solo un asunto del siglo XX, pues éste tiene ya una larga tradición en los últimos doscientos años, sobre todo si nos atenemos a los grandes pensadores de los albores de la independencia, a los grandes críticos liberales de mantener el antiguo orden hispánico, a los lìderes de los movimientos sociales de fines del siglo XIX e inicios del XX, a los grandes críticos de la celebración del primer centenario, y en particular, varios filósofos que emprendieron una reflexión cuestionadora del orden de cosas existente en Chile.

Atendiendo a esos antecedentes podría postularse la hipótesis que, considerando en general el pensamiento crítico, éste ha sido una parte decisiva del pensamiento nacional, ya que la dimensión reflexiva y cuestionadora se encuentra en muchos pensadores. En este trabajo nos abocaremos solamente a tres pensadores que han contribuido poderosamente al conjunto del quehacer filosófico en Chile durante el siglo pasado, iniciando con ellos una línea investigativa y aplicándola luego luego a otros filósofos chilenos. Los tres elegi-

\footnotetext{
Gandler, S., "Reconocimiento versus ethos", en Íconos. Revista de Ciencias Sociales, No 43, (2012) pp. 47-64.

2 Se pueden citar cuatro obras relevantes: Dussel, E. \& Otros, El pensamiento filosófico latinoamericano, del Caribe y 'Latino' (2011), Tealdi, Diccionario de Bioética, (2008), Biagini y RoIg, Diccionario de pensamiento alternativo (2008), y Salas, R., Pensamiento Crítico Latinoamericano (2005).
} 
dos tienen que ver con nuestro vínculo con algunos de sus textos y/o comentaristas y con el desarrollo de sus respectivos pensamientos a lo largo del siglo XX, marcando en buena parte el debate intelectual, generando debates en la opinión pública y otros contextos más especializados, ayudando todos ellos a renovar los estudios filosóficos en un sentido profesional, fecundando el amplio campo de las humanidades. Abre nuestra trilogía E. Molina, un filósofo que está en el intersticio del siglo, y cuyo impacto se sintió en la primera mitad del siglo XX. Seguiremos con J. Millas, quien elabora su pensar en la mitad del siglo pasado, y terminaremos con H. Giannini, cuya producción se concentra en el término de siglo.

Ciertamente deberíamos estudiar otros casos en función de realizar un trabajo plenamente exhaustivo, sin embargo, solo queremos aportar con una línea de trabajo que destaque y promueva otro sentido más fuerte del pensar filosófico, un pensar entendido no solo como estudio de las ideas, esto es, como exégesis de textos. La idea es buscar su enraizamiento en tradiciones que son parte de un mundo construido por el (los) filósofo(s), que apuntan no solo a una "emancipación mental", como se dijo en otras épocas, sino también a búsquedas socio-históricas de los países de liberación político-cultural.

Lo que buscamos argumentar aquí es que esta forma especializada de hacer filosofía en estos autores no disminuye de ningún modo su talante reflexivo y crítico frente a las formas inauténticas o ideológicas de hacer filosofía. En ellos se encuentra un pensar reflexivo y crítico que se asume como una práctica vigilante de lo que acontece en el país. Esta hipótesis, aunque en general es aceptable, plantea quizás una serie de reservas sobre todo en algunos sectores culturales, y no solo de filósofos "academicistas", en quienes la noción de crítica ha perdido su mordiente cultural. Algunos profesores todavía analizan los sistemas filosóficos como si ellos fueran parte de un proceso de ideas extemporáneas, justificando un ejercicio intelectual de aclaración de categorías que valen por sí mismas. Esto se mezcla con el canon incuestionable de los filósofos reconocidos, en especial si se trata de aquellos promovidos por la historia europea de la filosofía.

Desde nuestra óptica, la crítica no es un ejercicio epistémico, sino una cuestión éticopolítica de lo socialmente existente que presupone tanto un cuestionar la mera facticidad como también un cierto horizonte ideal hacia donde un país o una sociedad puedan transitar. Sabemos que ya existen algunas obras generales del pensamiento crítico y alternativo, de hecho por cada uno de los países de la región ya aparecen estudios acerca de las obras de los pensadores críticos de cada país, de las tradiciones que conforman y forman parte ese grupo de los llamados pensadores rebeldes (Jaksik). Este pensar reflexivo y crítico, tal como se ha elaborado desde el contexto europeo de la Ilustración, se trata de un pensar preocupado por alcanzar la mayoría de edad, sin tutelas y sin dogmatismos, cuestionando los presupuestos de los sistemas de dominación y disciplinamiento. Pensar críticamente, en definitiva, plantea la cuestión de la emancipación económica, política y cultural de un país, pero sin dejar de notar que, en este caso, la Ilustración europea es solo un inicio provinciano de la crítica de sus propias tradiciones. 


\section{¿Qué entender por una historia de las ideas?}

La propuesta de una historia de las ideas en Latinoamérica tiene una larga trayectoria en estas últimas décadas. En tal reconstrucción histórica del pensamiento filosófico latinoamericano se han hecho conocidas las propuestas sistemáticas de L. Zea, A. Roig, A. Guy, entre otros, quienes intentan comprender en toda su complejidad y amplitud las ideas que se elaboraron en el marco de diferentes corrientes y tradiciones sedimentadas en la compleja historia de los países iberoamericanos. Una de las principales conclusiones a que llegan es que las ideas de los pensadores de Iberoamérica son parte de una producción filosófica contextualizada desplegada en diferentes tradiciones y escuelas que responden a los desafìos histórico-políticos vivenciados por sus respectivos países. Corrientes tales como el positivismo, el liberalismo, el socialismo y el conservadurismo católico cumplieron funciones sociales y políticas de primera importancia. Lo que interesa es demostrar que no solo se trató de copiar ideas pensadas en otros contextos, que tampoco era solo un asunto de aplicación de ideas, sino que en muchos casos se debatían nuevas cuestiones, se hacían otros énfasis, etc. Esta etapa de estudios de las fuentes y recuperación de la obra de muchos autores ha avanzado sustantivamente, aunque todavía quedan pensadores y pensadoras desconocidas y anónimas. Dicho proyecto de búsqueda en nuestro propio pasado intelectual ha seguido un curso sistemático, lo que es parte de la meta de los fundadores de los estudios de las ideas: la propuesta de los primeros estudiosos se ha ido cumpliendo paulatinamente y hoy podemos contar con personas dedicadas plenamente a estudiar este pensamiento filosófico chileno.

Por todo lo dicho, entenderemos la historia del pensamiento filosófico en el Chile del siglo XX a partir de una comprensión general de las ideas en su intersección con los universos sociales, y no solamente como ideas filosóficas en un sentido técnico de la palabra. Por ello, manejaremos aquí la tesis según la cual las ideaciones no son solo elaboraciones subjetivas sino que son, de algún modo, vectores de construcción de las realidades sociales, culturales y políticas de los países ${ }^{3}$.

Empero caben algunas consideraciones metodológicas en el caso de Chile. Sabemos que el estudio de las ideas estuvo, desde inicios del siglo XX, asociado con frecuencia meramente al quehacer historiográfico (Donoso, Astorquiza, Hanisch). Además, hay que notar que a veces los propios filósofos profesionales -salvo honrosas excepciones- no destacaron en su estudio ni en la profundización de lo que representaban dichas ideas para las formas presentes que asume el quehacer filosófico. Salvo ilustres casos, los curriculum filosóficos no entregaban una visión de conjunto de la filosofía chilena del siglo XX, ni se

3 En este sentido, es señera la propuesta de una historia de las ideas como la vienen trabajando algunos colegas y amigos a nivel latinoamericano y que generan espacios de encuentros como Solar, Corredor de las Ideas y otros que abren pistas novedosas al tratamiento de las ideas, no solo en relación a los procesos ideacionales nacionales, sino también a los vinculaciones internacionales de estas ideas en procesos de construcción de un plural pensar latinoamericano. 
estudiaron las ideas de los grandes temas de los pensadores chilenos. Todo parece indicar que la filosofìa en Chile es una disciplina con asignaturas moduladas por el pensar europeo y norteamericano.

Estudiar de esta manera las ideas de pensadores chilenos del pasado que no formaban parte de las grandes ligas filosóficas europeas era, por lo general, considerado por los historiadores un capítulo del estudio de las élites, de los orígenes de los partidos políticos o de los movimientos sociales que no merecían la atención de los profesionales de la filosofía, quienes únicamente favorecían el estudio de los grandes filósofos de la Antiguedad y de la Modernidad europea. Así, es frecuente encontrar en las grandes interpretaciones elaboradas por el amplio abanico de los historiadores chilenos -que podría ir desde Diego Barros Arana, Benjamín Vicuña Mackenna hasta Gonzalo Vial, Gabriel Salazar y Sergio Villalobos- referencias acerca de las ideas de algunos grandes intelectuales que han marcado la historia del pensamiento nacional. Pero la investigación de las ideas no es solo cuestión de los historiadores, sino de estudiosos del pensamiento, y dentro de este vasto campo, de los estudiosos del quehacer de los sistemas filosóficos.

Por otra parte, en ciertos países latinoamericanos tales como México, Colombia, Ecuador, Perú y Argentina existen desarrollos notables de la comprensión de sus tradiciones filosóficas en ciertos periodos de su historia. Asimismo, en aquéllos encontramos permanentes reediciones de las obras del pasado, análisis e interpretaciones eruditas y estudios creativos de proyección de los textos y trabajos de los pensadores y filósofos del siglo XX. En Chile, en cambio, existe una enorme deuda y a la vez un gran desafío en cuanto a la necesidad de publicar los textos completos de estos nuevos modos de filosofar.

Con todo, un hito relevante ocurre en el Chile de los años setenta del siglo pasado, donde por diferentes razones se inicia un intento más o menos sistemático de intelectuales -con motivaciones muy distintas- que escudriñan las ideas críticas de pensadores nacionales en sus fuentes, comparando autores, sistematizándolos y situando estas ideas en el contexto pasado y presente que vive el cambio del país. Recientemente, han surgido intelectuales y jóvenes investigadores que han comenzado a analizar en forma sistemática las obras de los pensadores nacionales. No está demás volver a destacar los esfuerzos de Eduardo Devés, José Jara, Carlos Ossandón, Cecilia Sánchez, Bernardo Subercaseaux, quienes, con diferentes trayectorias, amplitud y profundidad, se han dedicado a estudiar esta rica veta del pensamiento crítico nacional. No está de más decir que estos nuevos enfoques de historia de las ideas tendientes a re-definir la filosofía en Chile permiten clarificar hoy el trasfondo filosófico de una tradición intelectual que durante mucho tiempo se la ha desconocido e invisibilizado en la formación universitaria. 


\section{¿Qué entender por un pensar reflexivo y crítico ?}

En algunos sectores de la filosofía contemporánea el pensar crítico puede parecer algo desfasado, en algún sentido anacrónico, sobre todo por corrientes culturales definidas por el des-encanto o por un pensamiento débil o postmoderno. Aunque puede parecer controversial, nos parece que justamente este ambiente cultural es el que clama por avanzar en una profundización de la reflexión y del pensamiento crítico. En esto seguimos una propuesta conocida en cierta tradición cosmopolita, que sostiene que la actividad filosófica no es aceptada no solo por religiones fundamentalistas y estados autoritarios, sino también por un cierto neoliberalismo de caracter modernizador. Esta perspectiva es ciertamente unilateral, ya que la filosofía en un sentido amplio es la única que permite mantener un pensar vigilante hacia procesos sociales y culturales que no esperan ser cuestionados por quienes detentan el poder. No es paradójico insistir entonces que, donde prima el quehacer filosófico, se abre siempre la posibilidad de jalonar procesos de mayor libertad social y de mayor igualdad para los mayorías.

Referirnos al pensamiento crítico requiere explicitar tres sentidos que parecen confundidos. En primer lugar, cabe precisar que todo pensamiento cuestionador requiere explicitar las formas de justificación de su propia aproximación, se trata de asumir lo que implica la producción intelectual en su contexto institucional de enunciación. En segundo lugar, este adjetivo también llama la atención respecto de problemáticas multidisciplinarias y de cruces categoriales donde la filosofía chilena aparece en un cruce epistemológico entre la filosofía, las ciencias sociales y humanidades. Pero, en el último sentido, se trata de ser crítico en oposición a un pensar que quedaría atrapado en los muros de las universidades e impide que florezca en el acompañamiento de los eventos históricos. Es, por ende, un pensamiento contestatario a las formas de ideologización.

No se trata, entonces, de contraponer en el caso de la filosofía chilena, tres autores relevantes que se caracterizan por "un pensar crítico" a otros pensadores en los que primarían las formas "ingenuas" de aprehensión de la realidad socio-cultural. Desde nuestra perspectiva, se trata de pensar una diferencia en la producción filosófica, entre los que piensan el mundo y los que se contentan con una mirada especializada de los textos. Por ello, en el ejercicio que devela las limitaciones de nuestra sociedad, estos tres filósofos chilenos que nos llaman la atención aquí, tuvieron y tienen conciencia de sus propias perspectivas disciplinarias y no solo apuntan a ejercer un cierto control y validación de las categorías filosóficas utilizadas por el trabajo intelectual, sino que, más allá del trabajo erudito, hay un horizonte intelectual ligado a la situación que vive el país. En este sentido, la hipótesis central es demostrar cómo la obra de autores como Molina, Millas y Giannini, junto con entregarnos lo más erudito de un pensar filosófico clásico, ofrecen una serie de pensamientos filosóficos donde manifiestan lo mejor de su pensar, teniendo una clara conciencia y amplia visión de los problemas sociales y políticos del país en que ellos tuvieron un relevante protagonismo. En este sentido, queremos demostrar una tesis donde la filosofía chilena como pensamiento crítico se identifica con esta perspectiva en que la filosofía no 
trepida en abrirse a los complejos contextos del país, dejando de ser un mero ejercicio de textos sobre textos.

Por consiguiente, en el significado fuerte del pensamiento crítico, hay un proceso reflexivo y crítico en los sujetos y movimientos sociales que no aceptan y cuestionan la hegemonía de un pensamiento autoritario, o como se indica hoy, un pensar que se propone como único y hegemónico, el cual puede ser el positivismo, el panamericanismo, el autoritarismo o el neoliberalismo. En suma, entendemos a la filosofía chilena en tanto pensamiento crítico del siglo XX cuando ésta despliega el sentido que transmite la propia reflexividad de la cultura y de la sociedad chilenas y aparece íntimamente relacionada con la historia, la política, la literatura y el arte.

En este sentido, entendemos a la filosofía chilena -en este breve esbozo históricocomo la permanente crítica de las teorías, cosmovisiones y formas de pensar que impiden el diálogo, la crítica y la comunicación. Pensar críticamente no es algo exclusivo de la filosofía, se da también en otras formas de racionalidad presentes tanto en Chile como en otros países latinoamericanos, donde la crítica, la protesta, la resistencia se han hecho patentes a lo largo de nuestra historia; lo propio de la filosofía es llevarlo a la máxima claridad gracias a un trabajo conceptual y argumentativo. Empero estos estudios sobre filosofía chilena se quedarían truncos sin considerar la inserción de las obras y de los pensadores nacionales en el marco general del pensamiento latinoamericano. Tal como lo he indicado en otros trabajos, es de capital importancia demostrar que las ideas no se ajustan a las fronteras definidas por los Estados, sino que operan con lógicas propias que tienen otras formas de difusión e irradiación al interior de redes intelectuales cada vez más amplias, como lo ha demostrado, por ejemplo, Eduardo Devés.

\section{Una hipótesis del pensamiento crítico en tres filósofos chilenos}

Hace algún tiempo, proyectando algunos nombres para la obra de E. Dussel, Mendieta y Bohorquez hicimos una encuesta a intelectuales de diferente disciplinas acerca de qué pensadores parecían más relevantes en este último siglo. No tuvimos una respuesta definitiva, pero existía cierta coincidencia en algunos autores que han marcado este siglo XX, existiendo ciertamente otros a los que podría incorporarse a este listado de pensadores críticos. Para establecer si un filósofo es crítico hay que precisar criterios que den cuenta del valor reflexivo de su obra, del impacto efectivo de sus ideas en la sociedad chilena y de las consecuencias de su pensamiento para el despliegue de otras formas de filosofía posteriores. La relevancia del aporte de otros filósofos más allá de los tres mencionados exige definir otros criterios para dar cuenta del hilo conductor de lo que acontece en el pensar crítico del siglo XX. Siguiendo las indicaciones ya señaladas en la hipótesis anterior, postulamos que una línea de pensamiento crítico - tal como lo entendemos acá- se podría encontrar en la obra y en el pensar de E. Molina, J. Millas y H. Giannini. 


\section{Enrique Molina (1871-1964)}

El filósofo Enrique Molina está ligado principalmente a la Región del Bio-Bío, a la fundación del Liceo de Chillán y de Concepción, y por cierto, a su enorme trabajo por construir una de las principales propuestas universitarias durante el siglo XX, a saber, la Universidad de Concepción. Empero, aún siendo E. Molina el artífice de un proyecto universitario regional, es un intelectual de relieve nacional e internacional, habiendo tenido un rol decisivo en pensar reflexiva y críticamente sobre la educación en general y, sobre todo, en pensar la universidad en el marco de los procesos que vivía la sociedad chilena de la primera mitad del siglo XX. En el terreno filosófico es uno de los primeros filósofos profesionales, poseedor de la mayor producción escrita de su tiempo. Fue primer presidente de la Sociedad Chilena de Filosofía y uno de los filósofos académicos más prolíficos en la escritura de textos filosóficos, los cuales le dieron el reconocimiento tanto en Chile como en el extranjero. En ellos mostró un caracter crítico ante la influencia de la filosofía positivista, abriendo el pensar a una vertiente humanista y espiritualista. Molina no solo es un intelectual universitario formado en la crítica especializada, sino que polemiza con las ideas tradicionalistas que aparecen en boga en la opinión pública y en el sentido común provinciano. En el conocido libro La herencia moral de la filosofía griega de 1936, muestra que la cuestión principal es la ética en su sentido teórico y práctico, ya que no solo se trataba de estudiar el legado de los conceptos griegos y sus principales valores (justicia, libertad y democracia), sino del modo como se podían aplicar a nuestro país. A este carácter ético de su obra se une una profunda comprensión del ser humano, pensar antropológico que estuvo unido en su persona a una perspicacia psicológica presente en todos los medios de prensa en que participó; empero, su crítica no ocultó su caracter de polemista.

Este auténtico pensador chileno, en su paso por Chillán y Concepción, tuvo una intensa actividad de prensa, donde muestra un talante progresista, liberal y librepensador. Miguel Da Acosta, uno de sus biógrafos y estudiosos más connotados, sostiene acerca de estos primeros artículos publicados por Molina en la prensa de Chillán y de Concepción:

La penetración psicológica que hace de algunos prototipos humanos, la crítica de costumbres anticuadas y, en especial, a la obstinada renuencia existente en algunos destacados personajes de la dirigencia ciudadana de Chillán, por no querer aceptar los nuevos presupuestos de la ciencia moderna son, en general, los temas que aborda con extraordinaria sagacidad ${ }^{4}$.

En el plano socio-político también se expresó el carácter crítico del pensar de Molina. En un artículo de 1925, por ejemplo, mantuvo profundas reservas acerca de la doctrina del Presidente norteamericano Monroe que pretendía defender América para los norteameri- 
canos, ya que la consideraba como un instrumento de colonialismo y vasallaje de Estados Unidos con respecto a los pueblos del sur, en un momento en que recién se conocían las ideas antiimperialistas del uruguayo Rodo. La actualidad de esta crítica de la idea de la influencia de los Estados Unidos en toda América, se expresa con mucha claridad en el cambio de la divisa de "América para los americanos", pues Molina prefiere el lema "América para la humanidad”. Nuestro autor se la jugó por la integración de Hispanoamérica, y planteó la idea de un nacionalismo solidario consistente en evitar los individualismos nacionales extremos que solo producen guerras entre países hermanos, con consecuencias desastrasas para los pueblos afectados. Mucho antes, en un ensayo titulado Ideales Sociales, analizaba los acontecimientos históricos de la Independencia con un prisma claramente filosófico y que se asentaba en un fino análisis basado en los conceptos de "patriotismo" y "paz". Al fin de la dictadura de Carlos Ibáñez dio su apoyo decisivo a la democracia, al expresar con argumentos filosóficos su resistencia a los regimenes totalitarios.

Otro tema ligado con su crítica al panamericanismo de Monroe, es su apasionada defensa de la cultura americana en su sentido más estricto, por una parte frente a una corriente que propugnaba romper violentamente con lo que se llama "vasallaje cultural europeo", privilegiando únicamente los valores de una perdida cultura autóctona; y, por otra parte, frente a otra corriente que intenta explicar la cultura americana como una simple localización prolongativa de la cultura europea.

\section{Jorge Millas (1917-1982)}

En un reciente libro ${ }^{5}$, Maximiliano Figueroa destaca con mucha fuerza el pensar comprometido y vigilante presente en la obra filosófica de Jorge Millas. Se trata de un libro de filosofía no porque alude a las peripecias de un filósofo, sino porque desenvuelve las principales ideas y argumentos que se esgrimieron en los tiempos de profunda transformación social y política de Chile, particularmente entre los años 1960-1980. En este período se construye el pensar crítico de este filósofo, el que le permite mostrar otra mirada para entender los conflictos de ese tiempo a partir de las ideas en pugna. En los últimos años de vida de Jorge Millas, entre 1973 y 1982, fue una de las pocas voces intrépidas y discordantes que se atrevieron a analizar en profundidad los problemas que vivía la sociedad chilena y ofrecer un testimonio de las consecuencias de un pensar a contracorriente, en tiempos que muchos intelectuales optaron por el silencio y la sumisión.

En este sentido, el pensar crítico y vigilante de Millas permite entender con mayor cabalidad las ideas en controversia en años marcados por la gran politización y crisis de la sociedad chilena y que se hizo eco sobre todo en los movimientos estudiantiles universitarios. Estos textos de Millas ofrecen una lectura de los fenómenos sociales que afectaron las universidades chilenas y muestran un cuadro de la mirada que la universidad tiene

5 FigueroA, Maximiliano, Jorge Millas.El valor de pensar, Universidad Diego Portales, Santiago, 2011. 
de ella misma. Pero, sobre todo, este pensar solitario de Millas da cuenta de la evolución de las ideas en los últimos dos gobiernos democráticos, desplegando un preciso análisis de la intervención militar que impuso una mordaza al pensamiento libre y crítico de sus académicos. En estos textos se observa a un académico prestigioso abogando por la racionalidad, el diálogo y el respeto de las personas, dejando en evidencia el bajo espesor moral de los interventores militares de la universidad.

El rico material que trae el libro de Figueroa -heterogéneo y a veces inédito-permite entender el desarrollo de un pensamiento crítico ligado a los contextos de la "reforma" en los tiempos de los presidentes Frei y Allende, y a la "intervención militar de la universidad". En ambos procesos queda en evidencia la valentía de un pensador discordante con las ideas masivas en boga entre los intelectuales y los estudiantes.

A través de la construcción del pensar vigilante de Millas, el autor intenta entender cómo la filosofía puede aportar con su instrumental analítico y crítico a entender la situación de la universidad en la complejidad sociohistórica de su tiempo, entregando argumentos para distinguir la filosofía de la ideología. Dotado desde joven de una notable capacidad oratoria, Millas llevará esta capacidad a pensar con sentido humanista crítico y reflexivo los grandes temas que afectan a la sociedad actual y a la universidad, la institución que debe promover el saber más riguroso.

Respecto de la tarea del intelectual, Millas postula que su misión es despertar a las gentes de una existencia sonambúlica. En un texto escrito para el premio R. Latcham indica que el escritor exhorta a los individuos a pensar por sí mismos para que se dispongan de un modo más lúcido y activo frente a la vida. Según Millas, se trata de construir una sociedad reflexiva, es decir "una sociedad que asuma la historicidad propia como condición exigida por la deliberación pública que ha de guiar la definición del destino". En este pensar crítico no solo cuestionará las ideas del marxismo ideológico por su dogmatismo, manifestado en un grupo de elegidos que creen tener el secreto de la situación histórica, sino también a las ideas antimarxistas que esconden a los detractores de la democracia.

Otro tema central que cruza desde muy temprano el pensar de Millas es el de la relación entre filosofía y violencia. En su defensa del pacifismo en el año treinta y ocho y sobre todo en su Idea de la Filosofía (1970), la filosofía es presentada como alternativa crítica a la posibilidad de la violencia y al sometimiento intelectual que los discursos ideológicos suelen implicar. Para Millas, para comprender la filosofía de la violencia hay que partir desde el dolor y sufrimiento de las víctimas. La violencia pone en relación lo fáctico y lo ético en el curso de la historia, y muestra las contradicciones a la que es llevado el militante de una causa revolucionaria por la que se infringe violencia y sufrimiento a otros.

Por otra parte, la democracia - para Millas- no representa solo un sistema de valía procedimental, sino que es, de un modo fundamental, un regimen con implicancias sustanciales, esto es, un auténtico proyecto político que compromete la construcción moral 
de la sociedad. En la obra ya citada, M. Figueroa destaca tres aspectos esenciales de la democracia: como forma de convivencia, como desideratum y como riesgo. Los derechos humanos constituyen una de las condiciones de posibilidad para la propia realización de la sociedad democrática.

Desde muy temprano, Millas advierte que el neoliberalismo de Hayek se construye a partir de deficiencias éticas y teóricas, criticando el concepto de libertad como ausencia de coerción, lo que implica cuestionar la idea del mercado como garante sin más de la libertad. Por esta vía las ideas del pensador austríaco hacen una incompleta consideración de la libertad humana: para entender adecuadamente la libertad es menester profundizar la naturaleza humana como tal, las particularidades individuales que dimanan de ella y la existencia de la sociedad humana. A juicio de Millas, el error de Von Hayek es la intencionalidad ideológica: "No es la libertad la que sirve de medida al capitalismo, sino éste a aquella".

Finalmente, la idea y defensa de la universidad son los textos más interesantes desde la perspectiva de la experiencia vital de Millas. Se trata de entender que la universidad tiene una reflexividad previa que parte de su naturaleza propia y de su responsabilidad ante la sociedad. Como lo afirma Millas tajantemente: "Antes que nada la universidad tiene que ser baluarte del conocimiento inspirado en la verdad y en la libertad y regulado por la discusión crítica"6. La universidad tiene que asumir el desafio de una sociedad de masas, pero la universidad misma no puede masificarse. Por ello, Millas entiende desde muy temprano que en una sociedad de masas más individuos presionarán por tener participación en el poder y la conducción del destino social, pero eso no puede llevar a que la comunidad universitaria pierda las funciones de cada miembro, y de ahí el rechazo de Millas a que los estudiantes intervengan directamente en el gobierno universitario. Por ello Millas se opuso a la politización de la universidad, ya que ésta la desnaturaliza, poniendo en jaque la ciencia y la crítica.

El filósofo Millas no claudicará en su defensa del pensar filosófico crítico y riguroso, a contracorriente de las ideas de los movimientos sociales y políticos en boga, que apelan a deconstruir ideas no suficientemente reflexionadas. Se trata de un pensar vigilante que no es repetición ni mera asunción de lo que han elaborado otros filósofos en otros contextos. En otras palabras, cobra pleno sentido la idea de alguien que quiso ser "irremediablemente, filósofo", como lo destaca el prologuista Humberto Giannini. Ser "irremediablemente, filósofo" es captar la labor sistemática de un pensador que llevó a "todas partes su pensamiento vigilante, insobornablemente reflexivo y ético por el que se caracterizó toda su vida"

6 Figueroa, Maximiliano, Jorge Millas.El valor de pensar, op. cit., p. 203.

7 Idem., p. 17. 


\section{Humberto Giannini}

H. Giannini es uno de los filósofos más conocidos de este tiempo, tanto en el medio universitario como en el grueso público común y corriente, no solo por la publicación de una Historia de la Filosofía que ha formado a muchas generaciones de jóvenes chilenos, sino porque su obra trasciende la elaboración de textos académicos, todos ellos con una honda resonancia en la situación que vivió y vive el país en los últimos treinta años. En el libro compilado por Cecilia Sánchez y Marco Aguirre titulado Humberto Giannini: filósofo de lo cotidiano queda en evidencia la potencia de un pensador de cuño existencial que se dedicó a pensar con rigor la autenticidad, la convivencia, la tolerancia, la reflexividad, el bien y la otredad en una serie de libros que han marcado a las nuevas generaciones de estudiantes de filosofía y que de algún modo han hecho escuela.

Entre los libros más importantes de Giannini se encuentra La reflexión cotidiana: hacia una arquelogía de la experiencia, que resume una categoría filosófica acerca de la re-flexión donde hay un espacio propio para lo propio y lo ajeno, y donde no solo cabe espacios para la intimidad como sería un talante existencial, sino también del encuentro y desencuentro con el otro, en el bar, la calle y la plaza. Cabe señalar que este libro no solo ha recibido elogios de Ricoeur, sino que ha inspirado trabajos creativos por parte de un grupo de sus discípulos.

Su compromiso con su reflexión filosófica le llevó a tomar posturas bien definidas en relación a la situación de la universidad en los tiempos del regimen militar, a sostener la defensa de los derechos humanos y el ideal de la democracia. Una frase de P. Vermeren puede retratar esta síntesis vital entre filosofía y compromiso con la enseñanza libre de la filosofía en la universidad pública: "A partir de la metafísica y de los conceptos que él se da sobre la tolerancia y la experiencia común, puede militar a favor de la educación pública y la enseñanza filosófica. Obstinadamente, desde la tribuna de su Cátedra de la Unesco de filosofía, donde repite también que la metafísica no ha muerto, su propia convicción de que la experiencia latinoamericana de la historia, de la naturaleza del otro, es una experiencia que importa esencialmente a la reflexión filosófica, y que los latinoamericanos pueden aportar una experiencia propia al problema de lo político"8.

Notable fue la decisión de Giannini de crear una sede independiente para su Departamento de Filosofía de la Universidad de Chile, emigrando a ella un selecto grupo de profesores comprometidos social y políticamente. Cabe recordar asimismo su gran preocupación por el pensamiento de Spinoza, dedicándole un número especial de la Revista de Filosofía, el cual fue vedado en su difusión por el régimen militar.

8 Sanchez, C. \& Aguirre, M., Humberto Giannini: filósofo de lo cotidiano, Lom-Universidad AHC, Santiago, 2011, p. 21. 


\section{Reconceptualizando el pensar crítico en el contexto histórico-cultural chileno}

Teniendo en cuenta algunos hitos temáticos de su peculiar manera de filosofar, sus diversas obras, así como los aportes y tópicos desarrollados por estos tres filósofos, hay que notar que la cuestión de la filosofía chilena como pensamiento crítico se ha logrado consolidar, dando origen recientemente a importantes direcciones teórico-prácticas. En todas estas diversas expresiones, más allá de sus innegables diferencias y matices como de los contextos históricos en que se desenvolvió el pensar de Molina, de Millas y de Giannini, intentamos responder a la cuestión de una filosofía comprometida con el mundo, y en todas ellos nos parece que la cuestión de la reflexión y la crítica surge como un tópico central para entender un nuevo modo de pensar la filosofía y comprender las vicisitudes propias de los contextos sociales y culturales de nuestros países. Por consiguiente, los tres filósofos responderían a la matriz de pensar crítico que propone este trabajo.

Empero, para que esta hipótesis sea demostrada ampliamente y pueda sostenerse como parte de una investigación de mayor aliento sería preciso estudiar si existen muchos más casos relativos a la filosofía chilena del siglo XX, y considerar si ella se puede ampliar al siglo XIX, e incluso abrirse a los tiempos anteriores a la constitución de la República. De hecho, se sabe que existen diversas tradiciones historiográficas y especulativas acerca de este pensar, tanto en la tradición histórica de los cronistas jesuitas expulsados del continente, -en la filosofía decimonónica-, como en las diferentes corrientes filosóficas de las primeras décadas del siglo XX. Sin embargo, daremos por presupuesta todas estas referencias a modo de un contexto general que permitiría hablar de una constante de la filosofía chilena y su sentido crítico.

No sabemos si en otros países se habrán dado con la misma intensidad las tensiones entre una filosofía académica universitaria y una propuesta en el marco de una filosofía latinoamericana, pero quisiera revelar que en el Chile de los años setenta-época en que estudiábamos-, preocuparse del "filosofar de autores chilenos" no tenía ningún lugar dentro del espacio académico formal, y para muchos jóvenes estudiantes que nos formábamos en la disciplina filosófica, esta búsqueda de un pensar impregnado desde los contextos histórico-culturales se debía realizar enteramente fuera de los cursos universitarios, lo que contrastaba fuertemente con lo que se vivía en otros países de la región. La cuestión de fondo es mucho más que una vicisitud personal, ya que la respuesta adecuada a un pensar filosófico crítico está enraizada claramente a los diferentes contextos sociales, culturales y políticos de Chile. En las últimas décadas se ha practicado este quehacer filosófico crítico en nuestras universidades y el sentido que se le asigna muestra una mayor preocupación por el pensamiento chileno crítico irrumpiendo éste al interior de los programas universi-

9 Una anécdota personal que puede ilustrar estos prejuicios academicistas es que estudiando Filosofía en los años ochenta, los estudiantes que hacían tesis de licenciatura en autores tales como A. Bello y E. Molina, eran blanco de las ironías de los académicos acerca de esa moda facilista de hacer tesis de filosofía en autores menores y que además escribían en castellano. 
tarios, pero casi siempre a la zaga de lo que acontece en otras universidades latinoamericanas, que ya llevan tiempo en estos menesteres.

La cuestión tratada exige una profundización respecto de las diversas formas que asumen las racionalidades al interior de las culturas, en la que es preciso destacar que el saber, la reflexión y la reconstrucción crítica surge por doquier en las diversas formas de entender el pensar filosófico. La filosofía chilena, en esta rápida panorámica filosófica del último siglo, nos permite entender en su plena complejidad el entramado del pensar, pero no como algo exclusivo de la filosofía, ya que el pensar requiere ampliarse al conjunto de las racionalidades presentes en las ciencias sociales y humanas, que de un modo u otro, han reflexionado, en un nexo entre explicación y comprensión, acerca de los paradigmas de la modernización, de la modernidad y de la identidad cultural, por mencionar tres temas relevantes de estos últimos años.

Pero la cuestión referida a la filosofía chilena como pensamiento crítico abre una interrogante más crucial, que me parece que a veces queda oculta entre los filósofos y los cientistas sociales. A veces existe una precomprensión de la racionalidad como algo exclusivo de las ciencias y de las racionalidades estructuradas a partir de una lógica científica: para decirlo de otro modo, hay un tipo de incomprensión acerca del espacio propio de la conciencia reflexiva y crítica en las culturas latinoamericanas, como si ella fuera algo que se reduce únicamente a los intelectuales profesionales. Justamente nuestra mirada apunta a cuestionar esta idea, de manera que un aporte del pensamiento crítico sería justamente ir a la inversa del modo practicado por las tendencias cientificistas y logicistas antes señaladas.

Por último, y como consecuencia de lo anterior, integraremos el pensamiento filosófico chileno de Molina, Millas y Giannini a un pensar filosófico latinoamericano que cuestiona las formas discursivas veladas o encubiertas, tan propias de las ideologías de la dominación, del cinismo imperante y de la negación del otro, que desgarran dramáticamente nuestros mundos vitales, pero que permiten, a pesar de estas limitaciones, la acción de los sujetos para transformar sus contextos y a veces reconstruir sus sueños de una segunda emancipación.

Mencionando estos diversos aportes y tópicos, queremos simplemente anotar que la cuestión de la filosofía chilena en estas tres décadas está dando origen a importantes vertientes especulativas y teóricas. En todas estas diversas expresiones, más allá de sus innegables diferencias y matices, se ha intentado responder de una forma rigurosa a la cuestión de una filosofía chilena como pensamiento crítico, y en todas ellas me parece que la cuestión de la reflexión y de la crítica surge como un punto central para entender un nuevo modo de pensar la filosofía en los contextos sociales y culturales de nuestros países latinoamericanos.*

*Artículo recibido: 27 de noviembre de 2012. Aceptado: 7 de marzo de 2013. 


\section{Bibliografía}

Astorquiza, Fernando, Bio-bibliografía de la filosofía en Chile desde el siglo XVI hasta 1980, Universidad de Chile, Facultad de Filosofia, Humanidades y Educación y el Instituto Profesional de Santiago, Imprenta Barcelona, Santiago, 1982.

Beorlegui, Carlos, Historia del pensamiento filosófico latinoamericano, Universidad de Deusto, Bilbao, 2004.

Bohorquez, Dussel, E. y Mendieta (Eds.), Historia del pensamiento filosófico latinoamericano, del Caribe y 'latino’ (1300-2000), CREFAL/Siglo XXI Editores, México, 2009.

Devés, E., El pensamiento latinoamericano en el siglo XX, Biblos-DIBAM, Buenos Aires-Santiago, 2003.

Devés, E. \& SAlas, R., “La filosofía en Chile (1973-1990)” en Cuadernos Hispanoamericanos, Madrid, (1991), pp. 71-80. Re-editado también en El pensamiento chileno en el siglo XX, FCE, México, 1999, pp. 199-212.

Escobar, Roberto, El vuelo de los búhos. Actividad filosófica en Chile de 1810 a 2010, Ril, Santiago, 2008.

Figueroa, Maximiliano, Jorge Millas. El valor de pensar, Universidad Diego Portales, Santiago, 2011.

Hanisch, Walter, En torno a la filosofía en Chile, Universidad Católica, Santiago, 1963.

JAKSIC, IvÁn, Academic Rebels in Chile, State University of New York Press, Albany, 1989.

Molina, Enrique, Obras Completas, Ediciones UDEC, Concepción, 1994.

Salas, Ricardo, "Introducción" a Pensamiento crítico latinoamericano, Ediciones UCSH, Santiago, 2005, 3 tomos.

----------- “Existe una filosofía latinoamericana?”, en Colodro, M., Foxley, A. M. y Rossetti, C., (Eds.), Palabra de Filósofo. Jornadas de reflexión en el Día Mundial de la Filosofía, Comisión Nacional UNESCO, Santiago de Chile, 2007, pp. 261-276. 
SÁnChez, CeCILIA, Una disciplina de la distancia. Institucionalización universitaria de los estudios filosóficos, CERC-CESOC, Santiago, 1992.

Sanchez, C. \& Aguirre, M., Humberto Giannini : Filósofo de lo cotidiano, LOM-Universidad AHC, Santiago, 2011.

Santos H., José, "Representaciones de la filosofía chilena en el siglo XIX”, en Vermeren P. Y MuÑoz M., Repensando el siglo XIX desde América Latina y Francia, Colihue, Santiago, 2009.

" "200 años: apuntes para una historia episódica de la filosofía en Chile”, en Revista Mapocho, 2010, pp. 323-352.

Subercaseaux, Bernardo, Historia de las ideas y de la cultura en Chile, Universitaria, Santiago, 4 tomos.

Vidal, Santiago, “Apuntes sobre la Filosofía en Chile”, en Revista La Cañada 3 (2012), pp. 254-271. 